1 Large protein complex production using the SmartBac System -

\title{
Strategies and Applications
}

3 Yujia Zhai ${ }^{1,2,{ }^{*}}$, Danyang Zhang ${ }^{1,2}$, Leiye $\mathrm{Yu}^{1,2}$, Fang Sun ${ }^{1,2}$, Fei Sun ${ }^{1,2,3,{ }^{*}}$

$4{ }^{1}$ National Key Laboratory of Biomacromolecules, CAS Center for Excellence in

5 Biomacromolecules, Institute of Biophysics, Chinese Academy of Sciences,

6 Beijing 100101, China.

$7{ }^{2}$ School of Life Sciences, University of Chinese Academy of Sciences, Beijing,

8 China.

$9{ }^{3}$ Center for Biological Imaging, Core Facilities for Protein Science, Institute of

10 Biophysics, CAS, Beijing, China.

11 * Correspondence should be addressed to Y.Z. (yujia@ibp.ac.cn) or F.S.

12 (feisun@ibp.ac.cn)

13 Keywords

14 Baculovirus system; Cre recombination; Gibson assembly; Vectors; Protein

15 complex production.

16

17 


\section{ABSTRACT}

2 Recent revolution of cryo-electron microscopy has opened a new door to 3 solve high-resolution structures of macromolecule complexes without

4 crystallization while how to efficiently obtain homogenous macromolecule 5 complex sample is therefore becoming a bottleneck. Here we report SmartBac, an

6 easy and versatile system for constructing large-sized transfer plasmids used to

7 generate recombinant baculoviruses that express large multiprotein complexes in

8 insect cells. The SmartBac system integrates the univector plasmid-fusion system,

9 Gibson assembly method and polyprotein strategy to construct the final transfer

10 plasmids. The fluorescent proteins are designed to be co-expressed with

11 recombinant proteins to monitor transfection and expression efficiencies. A

12 scheme of screening an optimal tagged subunit for effective purification is

13 provided. Six large multiprotein complexes including the human exocyst complex

14 and dynactin complex were successfully expressed, suggesting a great potential

15 of SmartBac for its wide application in the future structural biology study. 


\section{INTRODUCTION}

2 With the rapid development of single-particle cryo-electron microscopy

3 (cryo-EM), more and more macromolecular machineries structures, e.g.

4 spliceosome $e^{1,2}$, ryanodine receptor $^{3-5}$, anaphase promoting complex ${ }^{6}$, light

5 harvest complex ${ }^{7}$ and mitochondrial respirasome ${ }^{8,9}$, have been solved to

6 near-atomic resolution, which have been waiting for many years. Since cryo-EM

7 does not need crystals, many macromolecular complexes that are difficult to be

8 crystallized are now ready for structural studies; thus, more and more structural

9 biology laboratories are becoming interested in studying the structures of large

10 macromolecular complexes. However, how to obtain enough highly purified

11 specimen suitable for cryo-EM is therefore becoming a new bottleneck, which has

12 restricted the wide application of cryo-EM technology.

13 One method for obtaining large protein complexes is to extract them from

14 biological tissues. However, this method is not very suitable for low-abundance

15 samples. In addition to consuming large amounts of reagents, protein extraction

16 usually yields samples with low yield and purity. And even for high-abundance

17 samples, it is still difficult to prepare and purify mutant proteins for functional

18 studies. Thus, recombinant expression of protein complexes is more commonly

19 preferred.

20 The baculovirus expression system (BVES) is a powerful tool for recombinant

21 protein production ${ }^{10}$ because it is safe, high expression levels can be achieved,

22 and post-translational modifications can be incorporated. The most common

23 baculovirus used for gene expression is AcMNPV (Autographa californica multiple

24 nuclear polyhedrosis virus), which has a large, circular double-stranded DNA

25 genome (about 130kb) that can accommodate very large exogenous DNA 
1 fragments ${ }^{10}$. However, it is not easy to introduce foreign genes by conventional

2 molecular cloning methods due to the large size of AcMNPV genome. Therefore,

3 researchers have modified the AcMNPV genome to allow effective foreign gene

4 insertion by site-specific transposition or homogenous recombination ${ }^{11,12}$. The

5 widely-used Bac to Bac baculovirus expression system (Invitrogen Co.) is an

6 example of the successful use of this approach.

$7 \quad$ Three strategies are commonly used to overexpress multiprotein complexes

8 in insect cells. In the first strategy insect cells are infected with multiple types of

9 baculoviruses, each of which carries one or two gene expression cassettes

10 (GECs). This strategy, which involves molecular cloning, is relatively simple and

11 has been successfully applied to the Bac to Bac system by many research

12 groups $^{13-18}$. However, when multiple types of baculoviruses are used, the total

13 number of infectious viruses added to the expression culture should be

14 comparable to the total number added when the culture is infected with a single

15 baculovirus. This will inevitably lead to a lower protein yield. In addition, the

16 expression levels of the individual subunits are often imbalanced, which can result

17 in improper complex assembly.

18 The second strategy used to express multiprotein complexes is to construct a

19 transfer plasmid carrying multiple GECs. The commercial pFastbac-Dual vector

20 features two promoters for expression of two proteins simultaneously. Similar

21 triple or quadruple expression vectors have also been built using traditional

22 molecular cloning methods ${ }^{19}$. The MultiBac system generates multi-GEC donor

23 and acceptor vectors from junior plasmids carrying individual GECs by homing

24 endonuclease-based multiplication module ${ }^{20}$. Then the final transfer plasmid is

25 produced by Cre-mediated recombination between the donor and acceptor

26 vectors $^{20}$. Yet the problem of unequal subunit stoichiometry still exists. 
1 The third strategy is the polyprotein strategy that has been used by

2 coronaviruses to produce multiple functional nonstructural proteins (nsps), which

3 are involved in the formation of the replicase-transcription complex (RTC) that

4 mediates viral replication and transcription ${ }^{21}$. The nsps are encoded in

5 open-reading frame $1 \mathrm{a}(\mathrm{ORF} 1 \mathrm{a})$ and $\mathrm{ORF} 1 \mathrm{~b}$ and are synthesized initially as two

6 large polyproteins, pp1a and pp1ab. During or after synthesis, these poliproteins

7 are cleaved by virus-encoded proteases into $16 \mathrm{nsps}^{22}$. These nsps, together with

8 other viral proteins and, possibly, cellular proteins, assemble into

9 membrane-bound $\mathrm{RTC}^{23}$. This strategy has been exploited to produce protein

10 complexes in the baculovirus system in recent years ${ }^{24,25}$. By this strategy, idividual

11 subunits are separated by protease cleavage sites and expressed as a long

12 polyprotein. In vivo processing of the polyprotein allows the proper assembly of

13 the multi-subunit complex. This method is very good for balancing expression

14 levels and achieving the correct subunit stoichiometry ${ }^{24}$. But when expressing

15 high molecular weight multiprotein complexes, the DNA fragment encoding the

16 polyprotein is very long. It is usually not easy to build such large transfer plasmids

17 in the average laboratory. In addition, with increasing gene length, gene synthesis

18 becomes more expensive and time-consuming. Other potential problems include

19 instability of the recombinant baculovirus due to the large foreign gene insertion

20 and inefficient virus amplification.

21 There are several other considerations that need to be taken into account

22 when using BVES to express large multiprotein complexes. First of all, the

23 transfer vectors carrying genes for multiple subunits need to be designed so that

24 molecular cloning can be easily accomplished. Because the difficulty of molecular

25 cloning increases with the size of the target construct, transfer vectors that allow

26 efficient selection for positive transformants are necessary. Second, one tagged 
1 subunit protein is often used to purify entire protein complexes; however, due to a

2 lack of prior knowledge, sometimes we have to screen for the optimal tagged

3 subunit that allows the best purification of the entire complex. A smart

4 experimental scheme is needed to avoid exerting too much effort in building these

5 screening vectors. Third, it is also important to quickly determine whether protein

6 expression is sufficient and virus amplification is successful because insect

7 expression systems are more time-consuming compared with Escherichia coli

8 expression systems. The sooner these problems are identified, the faster they can

9 be solved.

10 To overcome these problems in expressing recombinant multisubunit proteins,

11 we have developed SmartBac, a simple and versatile vector system, which

12 combines the advantages of the three strategies described above. The SmartBac

13 vectors are optimized for building large constructs, and by using these vectors,

14 large protein complexes with an intricate subunit composition can be conveniently

15 expressed. The addition of a LacZ-alpha cassette allows positive recombinants

16 with large DNA inserts to be easily selected by blue-white screening. A univector

17 plasmid-fusion system (UPS) strategy has been incorporated in our vector

18 system $^{26}$, so that final construction of large transfer plasmids can be realized by

19 Cre-loxP site-specific recombination between donor and acceptor vectors. This

20 approach makes the preparation of large plasmids easier. To simplify vector

21 construction and obtain more homogenous samples, the polyprotein strategy and

22 Gibson assembly were used to construct the transfer plasmid. The co-expression

23 of EGFP and tagRFP in the SmartBac system provides real-time visualization of

24 transfection and expression. In addition, the SmartBac system can be used to

25 conveniently determine the best tagged subunit that provides the best purification

26 of the entire protein complex. Using the SmartBac system, we have expressed 
1 many large multiprotein complexes, including the human exocyst complex and

2 dyncatin complex. This system can also be applied to bacteria, yeast, or

3 mammalian cells with minor modifications. We expect the SmartBac system will

4 aid structural and functional studies of large multiprotein complexes in the future.

\section{RESUTLS}

\section{SmartBac Vectors}

7 In order to overcome the difficulties in building large plasmids with

8 conventional cloning methods, we used the broadly applicable UPS strategy ${ }^{26}$ to

9 set up our SmartBac vector system. Briefly, this strategy uses Cre-loxP

10 site-specific recombination to catalyze fusion between the univector (donor vector)

11 and host vector (acceptor vector). The kanamycin-resistant donor has a

12 conditional $\mathrm{R} 6 \mathrm{~K} \gamma$ origin of replication that allows its propagation only in bacterial

13 hosts expressing the pir gene, which encodes the essential replication protein

$14 \pi^{27,28}$. Selection for the UPS recombination products is achieved by selecting for

15 kanamycin resistance $\left(\mathrm{Kan}^{\mathrm{R}}\right)$ after transformation into a pir- strain; the Kan ${ }^{\mathrm{R}}$ gene

16 in the donor vector can be expressed in a pir- background only when covalently

17 linked to an acceptor vector that has a functional origin of replication (oriColE1) ${ }^{26}$.

18 This strategy has been successfully used in the MultiBac system ${ }^{20}$ and is

19 advantageous for the preparation of large plasmids and their mutants due to the

20 relatively small sizes of the donor and acceptor vectors.

21 We designed four acceptor plasmids (4V1G, 4V1R, 5V1TG and 5V1TR) and

22 two donor plasmids (4V2G and 4V2R) for use in the SmartBac system (Figure 1,

23 see also Supplementary Materials and Methods S1). The acceptors can

24 recombine with the donors via Cre-LoxP site-specific recombination. The

25 acceptors harbor a p15A origin of replication that allows propagation in common 
1 cloning strains of E. coli at low copy number, which is better for the stability of

2 large plasmids. The acceptors also contain resistance markers for ampicillin and

3 gentamycin and flanking mini-Tn7 elements for the generation of recombinant

4 baculoviruses.

5 Transgene expression in infected insect cells is driven by the baculovirus late

6 p6.9 promoter $^{29}$. Compared with the routinely used very late polyhedrin promoter,

7 the p6.9 promoter drives expression at earlier stages of infection when cells are

8 more likely to be in good condition and therefore the aggregation of expressed

9 foreign proteins may be avoided ${ }^{30-32}$.

10 The $4 \mathrm{~V} 1$ acceptor vectors $(4 \mathrm{~V} 1 \mathrm{G}$ and $4 \mathrm{~V} 1 \mathrm{R})$ carry an $\mathrm{N}$-terminal HA-tagged

11 TEV protease coding sequence followed by the TEV protease cleavage site (TCS)

12 and a Twin-Strep tag coding sequence followed by a recognition site for

13 enterokinase. Between multiple cloning site (MCS) 1 and 2, there is a LacZ-alpha

14 expression cassette, which allows blue/white selection of recombinant clones.

15 Downstream of MCS2, there is another TCS and an EGFP (4V1G) or TagRFP

16 (4V1R) coding sequence. The fluorescent and target proteins can be expressed

17 as a single ORF. By observing the fluorescence of infected cells, we can easily

18 determine whether the target protein has been expressed.

19 In the 5V1T acceptor vectors (5V1TG and 5V1TR), different from $4 \mathrm{~V} 1$

20 acceptor vectors, the TEV protease and EGFP (5V1TG) or tagRFP (5V1TR)

21 coding sequences are fused and expressed as a GP64 promoter-driven ORF.

22 The $4 \mathrm{~V} 2$ donor vectors $(4 \mathrm{~V} 2 \mathrm{G}$ and $4 \mathrm{~V} 2 \mathrm{R})$ carry an $\mathrm{N}$-terminal $10 \times$ His coding

23 sequence followed by a recognition site for enterokinase. Both vectors contain a

24 kanamycin resistance marker. The screening region is composed of a high-copy

25 PUC origin of replication and a LacZ-alpha expression cassette, flanked by MCS1

26 and MCS2. Downstream of MCS2, there is a TCS and a fluorescent protein 
1 (EGFP in 4V2G and tagRFP in 4V2R) coding sequence. The expression of the

2 target protein is driven by the very late $\mathrm{p} 10$ promoter. The $4 \mathrm{~V} 2$ vectors also

3 contain the conditional origin of replication, $\mathrm{R} 6 \mathrm{~K} \gamma$. Once the screening region is

4 replaced by a foreign gene, the donor vector only contains the R6K $\gamma$ origin and

5 can only be propagated in E. coli strains with the pir+ genotype.

6 There are several single restriction sites located on both sides of the p6.9 and

7 p10 promoter regions in the $4 \mathrm{~V} 1 / 5 \mathrm{~V} 1$ acceptor and $4 \mathrm{~V} 2$ donor vectors, 8 respectively, so that they can be replaced by other baculovirus promoters, if 9 needed.

10 Schemes for the expression of large multiprotein complexes using the

\section{SmartBac system}

12 The SmartBac system was designed for easier and faster expression of large

13 multiprotein complexes in insect cells. A variety of experimental schemes could be

14 applied to produce the final transfer plasmids from the SmartBac vectors. Here we

15 just present two schemes to exemplify how to use the SmartBac vectors. In the

16 example shown in Figure $\mathbf{2 a}$, a vector is designed to express a multiprotein

17 complex composed of eight different subunits (e.g. subunits $A, B, C, D, E, F, G$

18 and $\mathrm{H}$ ) in insect cells. If the molecular weight of the multiprotein complex is less

19 than $600 \mathrm{kDa}$, we propose using Scheme 1 (Figure 2a). The eight subunits are

20 divided into two groups so that the sum of the lengths of the genes in one group is

21 as similar as possible to the other group. Then for each group of genes, a fusion

22 DNA fragment (ABCD and EFGH) with TCS coding sequences separating the 23 adjacent genes is designed.

24 Next, the long $A B C D$ and EFGH fragments are further divided into two short

25 DNA fragments $A B$ and $C D$ and $E F$ and $G H$, which can be obtained easily by 
1 overlapping PCR (Figure 2b). To avoid unnecessary trouble in overlapping PCR,

2 the TCS cleavage sites described above should be coded by multiple degenerate

3 sequences (Supplementary Materials and Methods S2). Then, fragments $A B$

4 and $C D$ are assembled with a linearized SmartBac RFP-expressing acceptor

5 plasmid (we use 4V1R in Figure 2b, but 5V1TR can also be used) utilizing a

6 Gibson assembly reaction ${ }^{33}$. Fragments EF and GH are also assembled with a

7 linearized SmartBac GFP-expressing donor plasmid (4V2G) using the same

8 method. The positive recombinants can be easily selected by blue-white

9 screening. Finally, the acceptor-4V1R-ABCD and donor-4V2G-EFGH vectors are

10 recombined via Cre-LoxP site-specific recombination to generate the final transfer

11 plasmid ABCD-EFGH. After transforming this plasmid into $\mathrm{DH} 10 \mathrm{Bac}$ competent

12 cells, recombinant bacmid will be obtained. This bacmid will be transfected into

13 insect cells to produce high-titer baculovirus BV-ABCD-EFGH used to express the

14 target eight-subunit complex.

15 If the molecular weight of the multiprotein complex is greater than $600 \mathrm{kDa}$,

16 the size of the final transfer plasmid constructed using Scheme 1 will be larger

17 than $25 \mathrm{~kb}$. It is usually not easy to build such a large plasmid without experience.

18 And even if the construction is successful, the multiprotein complex may fail to be

19 expressed in insect cells. This is because the recombinant bacmid generated

20 from the large transfer plasmid is prone to display an intrinsic genetic instability

21 due to the large foreign gene insertion ${ }^{30,34}$. Spontaneous deletion of the foreign

22 gene insertion may occur during the amplification of P2 virus (our unpublished

23 data). So, in this case Scheme 2 should be used. As shown in Figure 2c,

24 Fragments $A$ and $B$ are assembled with linearized $5 V 1 T R$, and fragments $C$ and $D$

25 are fused with linearized $4 \mathrm{~V} 2$ vector. A stop codon has been inserted upstream of

26 the coding sequences by PCR so that the fluorescent protein in $4 \mathrm{~V} 2 \mathrm{G}$ and $4 \mathrm{~V} 2 \mathrm{R}$ 
1 will not be expressed. The same method is used to clone fragments $E, F, G$ and $H$.

2 Next, two different final transfer plasmids ABCD (RFP) and EFGH (GFP) are built.

3 These plasmids will produce two types of recombinant baculoviruses, one

4 expressing subunits $A, B, C$ and $D$ and RFP, and the other expressing subunits $E$,

$5 \mathrm{~F}, \mathrm{G}$ and $\mathrm{H}$ and GFP. Insect cells co-infected with both baculoviruses will produce

6 the entire protein complex, with the appearance of tagRFP and EGFP

7 fluorescence indicating successful expression of the target protein complex.

8 The SmartBac acceptors carry an optional N-terminal Twin-Step-tag 9 sequence, and the donors carry an optional N-terminal His-Tag sequence. Either

10 tag can be fused to a target subunit and used as a handle to purify the target

11 subunit along with its associated subunits. If the biochemical properties of the

12 protein complex are known, it is easy to determine which subunit is the most

13 suitable to fuse with the affinity tag. However, when previous knowledge is limited,

14 it may be hard to pick the appropriate subunit because different affinity-tagged

15 subunits often differ in their effectiveness in purifying the entire complex. Imagine

16 that we are expressing an eight-subunit complex and are not sure which subunit is

17 suitable for labeling. If we use a classical "Trial and Error" approach and construct

18 multiple large final transfer plasmids, the workload will be very high. In addition,

19 the complicated clone scheme is often confusing. To solve this problem, we

20 propose a simple and universal scheme (Scheme 3, see Figure 3). Two large

21 final transfer plasmids ABCD (RFP) and EFGH (GFP) are built according to

22 Scheme 2 but where none of the eight subunits are labeled with affinity tags

23 (Figure 3a). An additional eight smaller transfer plasmids (from V1-TSA to V1-TSH)

24 based on one acceptor vector (4V1G, 4V1R, 5V1TG or 5V1TR) are constructed,

25 each expressing an N-terminal Twin-Strep-tagged subunit. A total of ten

26 recombinant baculoviruses are obtained, including BV-ABCD (RFP), BV-EFGH 
1 (GFP) and BV-TSn (where $n$ ranges from $A$ to $H$ ) (Figure $3 b$ ). The insect cells are

2 co-infected with three types of baculoviruses, BV-ABCD (RFP), BV-EFGH (GFP)

3 and one type of BV-TSn. The baculovirus combinations used for screening are

4 shown in Figure 3c. After purification, we know affinity-tagged subunit $\mathrm{H}$ results in

5 the best purification of the entire complex. To increase yield and obtain a more

6 homogenous sample, a new intermediate vector containing tagged subunit $\mathrm{H}$,

7 EFG-TSH (GFP), is built (Figure 3d). The resulting new recombinant baculovirus,

8 BV-EFG-TSH (GFP), along with the existing recombinant baculovirus, BV-ABCD

9 (RFP), are used to express the multiprotein complex, and real-time infection and

10 expression is monitored by observing the fluorescence of co-expressed EGFP

11 and tagRFP.

\section{Multiprotein complexes expressed using the SmartBac system}

13 To test the SmartBac system, we expressed the human exocyst complex in

14 insect cells. The exocyst complex is responsible for tethering secretory vesicles to

15 the plasma membrane in preparation for soluble N-ethylmaleimide-sensitive factor

16 (NSF) attachment protein receptor (SNARE) mediated membrane fusion ${ }^{35}$. The

17 human exocyst complex contains eight evolutionary conserved subunits-EXOC1

18 (102 kDa), EXOC2 (104 kDa), EXOC3 (86 kDa), EXOC4 (110 kDa), EXOC5 (82

$19 \mathrm{kDa})$, EXOC6 (94 kDa), EXOC7 (78 kDa) and EXOC8 (82 kDa). Because the

20 published literature does not provide information about which subunit is the most

21 suitable for complex purification, we used Scheme 3 to screen the target subunits.

22 All of the vectors we built for exocyst expression are shown in Table 1 (see also

23 Supplementary Materials and Methods S3). First, we constructed two types of

24 recombinant baculoviruses, BV-E1547 and BV-E2863, to express the eight

25 subunits without any tags according to Scheme 2. These baculoviruses also

26 expressed tagRFP and EGFP, respectively, which allowed us to conveniently 
1 determine whether virus infection and protein expression was successful (Figure

2 4a). We also produced eight additional types of recombinant baculoviruses, each

3 expressing an individual subunit with an N-terminal Twin-Strep tag (BV-SE1 to

4 BV-SE8). Then we co-infected insect cells with BV-E1547, BV-E2863 and a

5 baculovirus expressing a single affinity tagged-subunit (BV-SE1 to BV-SE8). The

6 best purification of the entire exocyst complex was achieved using tagged EXOC5

7 (BV-SE5) (Figure 4b). Then we constructed a new donor vector 4V2-E1S5 which

8 contains EXOC1 and N-terminal Twin Strep-tagged EXOC5. Recombination

9 between 4V2-E1S5 and 5V1TR-E47 (contains EXOC4 and EXOC7) produced a

10 new final transfer plasmid E1S547 (Table 1), from which recombinant baculovirus

11 BV-E1S547 was obtained. Insect cells were co-infected with BV-E1S547 and

12 BV-2863. After one-step strep-affinity purification, the entire exocyst complex with

13 high purity was obtained (Figure 4c). The tethering activity of this purified exocyst

14 complex was determined via In vitro liposome reconstruction assay (data is not

15 shown here). Negative-staining electron microscopy (nsEM) of the sample

16 revealed homogenous rod-like particles (Figure 4d). Preliminary 2D classification

17 of nsEM images (Figure 4e) and 3D reconstruction (Figure 4f) indicate that the

18 human exocyst complex exhibits a similar dimension and shape with the extracted

19 exocyst complex from yeast ${ }^{36}$. The detailed information about primer design,

20 molecular cloning, cell transfection, protein expression and purification, and

21 electron microscopy is described in Supplementary Materials and Methods S4.

22 We also reconstituted the human dynactin complex using the SmartBac

23 system. Dynactin is a multiprotein complex that works with cytoplasmic dynein to

24 transport cargo along microtubules. It is a large complex of approximately 1.2

25 MDa composed of 23 subunits corresponding to 11 different types of proteins ${ }^{37}$.

26 Dynactin is built around a short actin-like filament composed of Arp1 (43 kDa, 8 
1 copies) and $\beta$-actin (42 kDa, 1 copy). The barbed end and the pointed end of this

2 filament are capped by the CapZ $\alpha$-CapZ $\beta$ complex (33 kDa, $31 \mathrm{kDa}$ ) and the

3 Arp11-p25-p27-p62 complex (46 kDa, 20kDa, 21 kDa, 52 kDa) respectively. The

4 shoulder complex, which is made up of p50 (45 kDa, 4 copies), p24 (21 kDa, 2

5 copies) and p150/p135 (142 kDa/127 kDa, 2 copies), is positioned toward the

6 barbed end of the Arp1 filament ${ }^{38}$. As shown in Table 2, three types of vectors

7 were used to express the 11 subunits of the dynactin complex, and the $\mathrm{N}$-terminal

8 Twin-Strep tag on p135 was used to purify the whole complex. The shoulder

9 complex proteins, p135, p50 and p24. were expressed by BV-M5, which was

10 generated from the plasmid 5V1TG-M5. The final transfer plasmid AB was

11 obtained through recombination of the acceptor plasmid 5V1TR-B and donor

12 plasmid 4V2-A. Plasmid $A B$ was then used to produce the recombinant

13 baculovirus BV-AB expressing the other eight dynactin subunits. Insect cells were

14 co-infected with BV-M5 and BV-AB to express the entire dynactin complex. After

15 one-step strep-affinity purification, the dynactin complex was purified well with

16 rational stoichiometry of its subunits (Figure $\mathbf{4 g}$ ). After glycerol density gradient

17 ultracentrifugation, the further purified dynactin complex exhibited a single visible

18 band on a native gel (Figure 4h), suggesting a high homogeneity of the specimen.

19 This band was excised and subjected to mass spectrometry and all 11 human

20 dynactin complex subunits were identified (our unpublished data). This recombant

21 human dynactin complex sample was further investigated by nsEM and

22 subseqeuent 2D image classification (Figure 4i), showing a rod-like particle with

23 a shoulder at one end, which is similar to that of the endogenous dynactin

24 complex purified from pig brains ${ }^{38}$.

25 Besides the human exocyst and dynactin complexes, we also successfully

26 expressed many other protein complexes using the SmartBac system (Figure 4j). 
1 These include the human COPI complex (7 subunits, $558 \mathrm{kDa})^{39}$, cytoplasmic

2 Dynein complex (12 subunits, $1380 \mathrm{kDa})^{40}$, CSN complex (8 subunits, $\left.343 \mathrm{kDa}\right)$

$3{ }^{41}$ and SCF complex (5 subunits, $\left.180 \mathrm{kDa}\right)^{42}$. The recombinant COPI complex

4 sample has been used to study the structure of coatomer in its soluble form ${ }^{39}$.

5 Our results indicate that the SmartBac system can be used to express a wide

6 range of large multiprotein complexes.

\section{DISCUSSIONS}

8 Obtaining large multiprotein complexes through recombinant expression has

9 always been challenging for researchers who need a sufficient quantity of

10 high-purity protein for structural or biochemical studies. The key to successful

11 protein production using the baculovirus expression system is the construction of

12 the final transfer plasmid containing multiple protein subunits. The MultiBac

13 system uses polycistronic vectors carrying multiple GECs for the expression of

14 multiprotein complexes ${ }^{20}$. This expression strategy requires junior plasmids

15 containing only one GEC to be constructed first. Then several rounds of plasmid

16 construction have to be done to obtain multi-GEC-contained donor and acceptor

17 vectors. The final transfer plasmid carrying all GECs is produced by Cre-LoxP

18 recombination between the donor and acceptor vectors. MultiBac has been

19 proven powerful in generating multiprotein complexes ${ }^{24,43}$, especially when

20 robotic support is available. But in ordinary laboratories without robotics, the first

21 two procedures require a great deal of time. And as more GECs are added to a

22 single vector, plasmid construction becomes more difficult, due to the increasing

23 size of the plasmid and the lack of an effective screening method for large positive

24 recombinants. 
1 To simplify the process for constructing vectors that express multiple protein

2 subunits, we developed the SmartBac system. We mimicked the polyprotein

3 production strategy of coronavirus to realize the expression of multiple subunits.

4 Although this strategy has been discussed and applied in some laboratories

$5 \quad 25,44,45$, a specialized vector system and standardized procedures are not available.

6 By generating one GEC expressing a long polyprotein composed of multiple

7 subunits in donor and acceptor vectors, the SmartBac system does not require

8 the construction of numerous junior vectors. Large numbers of gene fragments

9 with overlapping sequences can be produced rapidly by PCR and directly used for

10 Gibson assembly with linearized vectors. Positive recombinants can be easily

11 selected by blue-white screening and then donor and acceptor vectors carrying a

12 long GEC can be combined by Cre-LoxP recombination to produce the final

13 transfer plasmid.

14 To ensure a high success rate, we fused multiple gene fragments together by 15 overlapping PCR so no more than three DNA fragments (including the linearized 16 vector) were included in a single Gibson assembly reaction. The size of the final

17 transfer plasmid produced using the schemes we have provided is usually less

18 than $25 \mathrm{~kb}$, so either chemical transformation (Trans2blue or XL10-Gold

19 ultracompetent cells) or electroporation can be used.

20 To increase the stability of the large final transfer plasmid propagated in E. coli,

21 we included a p15A replication origin of low copy number in the acceptor vectors

22 and cultured the bacteria at $30^{\circ} \mathrm{C}$. The restriction sites flanking the promoter

23 region allow for promoter exchange if needed (eg. To optimize expression levels).

24 To monitor the expression of target proteins, we added the most commonly 25 used fluorescent proteins EGFP and tagRFP genes to the SmartBac vectors, so 
1 that observation can be easily performed with a basic fluorescence microscope.

$24 \mathrm{~V} 1$ and $5 \mathrm{~V} 1$ vectors provide two different expression modes for these protein

3 markers. $4 \mathrm{~V} 1$ vectors use a single GEC to express these fluorescent proteins,

4 TEV protease and the target protein subunits. However, for some constructions,

5 the fluorescent protein was insufficiently cleaved from its upstream expressed

6 subunit (our unpublished data). It had a bad effect on the assembly of the entire

7 complex. In this case, $5 \mathrm{~V} 1$ vectors can be selected because they use one GEC to

8 express the target subunits, and another GEC to express the fluorescent protein

9 and TEV protease. We have provided two easy-to-use schemes to guide the

10 design of large transfer plasmids containing multiple genes as well as a verified

11 scheme to screen an optimal tagged subunit for complex purification.

12 With the development of cryo-EM, more and more research groups are

13 carrying out structural and functional studies of multiprotein complexes.

14 Recombinant expression is a promising method for obtaining sufficient quantities

15 of high-purity samples, and, we expect that the SmartBac system will allow more

16 researchers to successfully express and purify large multiprotein complexes.

\section{COMPETING INTERESTS}

19 Parts of this study (SmartBac system) has been submitted to apply a Chinese

20 patent for invention with the application number of 201610248592.8 .

\section{AUTHORS' CONTRIBUTIONS}

22 Fei Sun initiated and supervised the project. YZ designed all the SmartBac

23 systems including vectors and application strategies. YZ performed all the 24 experiments of molecular cloning and expression constructs production. YZ, DZ, 
1 LY and Fang Sun performed protein complex purification and preliminary electron

2 microscopic characterization. $\mathrm{YZ}$ and Fei Sun wrote the manuscript.

\section{ACKNOWLEDMENTS}

$4 \quad$ We would be grateful to Ping Shan and Ruigang Su (Fei Sun's lab) for their

5 help on lab maintenance. We would like to thank Xiang Ding and Mengmeing

6 Zhang from Laboratory of Proteomics, Core Facilities for Protein Science, at the

7 Institute of Biophysics (IBP), Chinese Academy of Sciences (CAS), for their help

8 with mass spectrometry analysis. We would like to thank Center for Biological

9 Imaging (CBI, http://cbi.ibp.ac.cn), IBP, CAS for the electron microscopy work.

10 This work was supported by grants from the Strategic Priority Research

11 Program of Chinese Academy of Sciences (XDB08030202) to Fei Sun, the

12 National Basic Research Program (973 Program) of Ministry of Science and

13 Technology of China (2014CB910700) to Fei Sun, and National Natural Science

14 Foundation of China (31771566) to YZ. 


\section{MATERIALS AND METHODS}

\section{Vector construction}

3 A portion of the $4 \mathrm{~V} 1$ vectors was derived from pFastbacDUAL (Invitrogen,

$4 \quad$ USA). $4 \mathrm{~V} 2$ vectors and all other portions of the $4 \mathrm{~V} 1$ vectors were synthesized by

5 Genewiz, China. The DNA fragments were fused together via Gibson assembly

6 (E2611, NEB, England) to generate the 4V1G, 4V1R, 4V2G and 4V2R vectors.

7 Then, using $4 \mathrm{~V} 1$ vectors, $5 \mathrm{~V} 1$ vectors were generated by Gibson assembly and

8 other classical molecular cloning methods. The sequences of the SmartBac

9 vectors are shown in Supplementary Materials and Methods S1. We

10 recommend using SnapGene Viewer (http://www.snapgene.com/) to view the

11 plasmid maps.

\section{Gibson assembly reactions}

13 The linearized plasmid fragments were obtained by restriction enzyme

14 digestion or PCR using Q5 High-fidelity DNA Polymerase (M0492, NEB, England).

15 DNA fragments to be inserted into the SmartBac vectors were amplified by PCR

16 to produce the appropriate overlaps. The overlapping primers were designed

17 according to NEB instruction manual for E2611. Assembly was done in a 15-20 $\mu \mathrm{l}$

18 reaction volume with 0.2-0.3 pmols each DNA fragment. Samples were incubated

19 in a thermocycler at $50^{\circ} \mathrm{C}$ for 60 minutes.

\section{Blue-white selection of positive recombinants}

21 To perform blue-white selection, $2.5 \mu$ l assembled product was added to $100 \mu \mathrm{l}$

22 chemically competent cells. The 4V1- and 5V1-based constructs were

23 transformed into chemically competent Mach1 ${ }^{\mathrm{TM}}$-T1R (Invitrogen) or DH5alpha or

24 Trans2-blue (TransGen Biotech, China) cells. After $1 \mathrm{~h}$ recovery in SOC medium

25 at $37^{\circ} \mathrm{C}$, cells were plated onto LB agar plates containing $100 \mu \mathrm{g} / \mathrm{ml}$ ampicillin, 40 
$1 \mu \mathrm{g} / \mathrm{ml} \mathrm{IPTG}$ and $100 \mu \mathrm{g} / \mathrm{ml}$ Bluo-gal. The 4V2-based constructs were transformed

2 into chemically competent GT115 cells (InvivoGen, USA), and then cells were

3 plated onto LB agar plates containing $50 \mu \mathrm{g} / \mathrm{ml}$ kanamycin, $40 \mu \mathrm{g} / \mathrm{ml} \mathrm{IPTG}$ and

$4100 \mu \mathrm{g} / \mathrm{ml}$ Bluo-gal. Single white colonies were picked and grown in $5 \mathrm{ml}$ LB

5 medium with the proper antibiotics for further plasmid extraction and PCR

6 analysis. The positive recombinants were sequenced at BioSune, China.

7 Production of the final transfer plasmid by Cre-LoxP Recombination

8 The donor and acceptor vectors ( 0.1 pmols each) were mixed with $1 \mu$ Cre

9 recombinase (M0298, NEB) in a $20 \mu \mathrm{l}$ reaction and incubated at $30^{\circ} \mathrm{C}$ for $1 \mathrm{~h}$. Ten

10 microliters of the reaction mixture were added to $100 \mu$ l chemically competent

11 Trans2-blue cells. After heat-shock at $30^{\circ} \mathrm{C}$ for $30 \mathrm{~s}, 500 \mu \mathrm{SOC}$ medium was

12 added, and the suspension was incubated at $37^{\circ} \mathrm{C}$ for $1 \mathrm{~h}$ with shaking (if the size

13 of the recombined vector was larger than $15 \mathrm{~kb}$, the suspension was incubated at

$1430^{\circ} \mathrm{C}$ for $\left.4 \mathrm{hrs}\right)$. The cell suspension was plated on LB agar plates containing 50

$15 \mu \mathrm{g} / \mathrm{ml}$ kanamycin and $100 \mu \mathrm{g} / \mathrm{ml}$ ampicillin. The plates were incubated at $37^{\circ} \mathrm{C}$

16 overnight (or $30^{\circ} \mathrm{C}$ for $24 \mathrm{hrs}$ ). Positive colonies were verified by PCR using the

17 primers Loxp-F (5'-CCACTGCGCCGTTACCAC-3') and Loxp-R

18 (5'-GCCGGTATGTACAGGAAG-3'). A 375 bp PCR product was amplified from

19 positive clones. The final transfer plasmids were extracted from the positive

20 clones.

\section{Production of Recombinant Baculovirus}

22 Chemically competent $\mathrm{DH} 10 \mathrm{Bac}$ cells were transformed with the final transfer

23 plasmid according to the Bac to Bac manual instructions (Invitrogen). For

24 transformation of large plasmids, the transformation mixture was incubated at

$2530^{\circ} \mathrm{C}$ with shaking for $8-12 \mathrm{hrs}$ and plates were incubated at $30^{\circ} \mathrm{C}$ for more than 
$148 \mathrm{hrs}$. Single white colonies (3-4) were innoculated into $5 \mathrm{ml}$ LB medium

2 containing $50 \mu \mathrm{g} / \mathrm{ml}$ kanamycin, $14 \mu \mathrm{g} / \mathrm{ml}$ gentamicin, and $10 \mu \mathrm{g} / \mathrm{ml}$ tetracycline.

3 Recombinant bacmids were extracted and verified by PCR amplification with

4 three pairs of primers (Tn7R:5'- GTTTTCCCAGTCACGAC-3' and

5 5'-AAGTTTGAGCAGCCGCGTAG-3’; Tn7L:5’- 5‘-CAGGAAACAGCTATGAC-3’

6 and 5‘-ACCTCCCCCTGAACCTGAAA-3’; Empty: 5'-GTTTTCCCAGTCACGAC-3’

7 and M13 Reverse :5'CAGGAAACAGCTATGAC-3’). Using the "Tn7R" and "Tn7L"

8 primer pairs, PCR products of $661 \mathrm{bp}$ and $521 \mathrm{bp}$, respectively, are amplified from

9 recombinant bacmids. If the recombinant bacmid is contaminated with wild-type

10 bacmid, a PCR product of $300 \mathrm{bp}$ will produced using the "Empty" primer pairs. It

11 is recommended to verify the existence of all of the subunit genes in the

12 recombinant bacmids by $\mathrm{PCR}$ if the size of final transfer plasmid is larger than

$1320 \mathrm{~kb}$.

14 Transfection and Virus Production in Insect cells

15 Transfection and Baculovirus production were done according to the Bac to

16 Bac manual (Invitrogen, USA). Successful transfection was determined by the

17 expression of EGFP and/or tagRFP fluorescent proteins. P2 virus was used for

18 expression.

\section{REFERENCES}

211 Yan, C. et al. Structure of a yeast spliceosome at 3.6-angstrom resolution.

22 Science (New York, N.Y 349, 1182-1191, doi:10.1126/science.aac7629

23 (2015).

242 Nguyen, T. H. D. et al. Cryo-EM structure of the yeast U4/U6.U5 tri-snRNP at

253.7 A resolution. Nature 530, 298-302, doi:10.1038/nature16940 (2016).

263 Yan, Z. et al. Structure of the rabbit ryanodine receptor RyR1 at near-atomic 


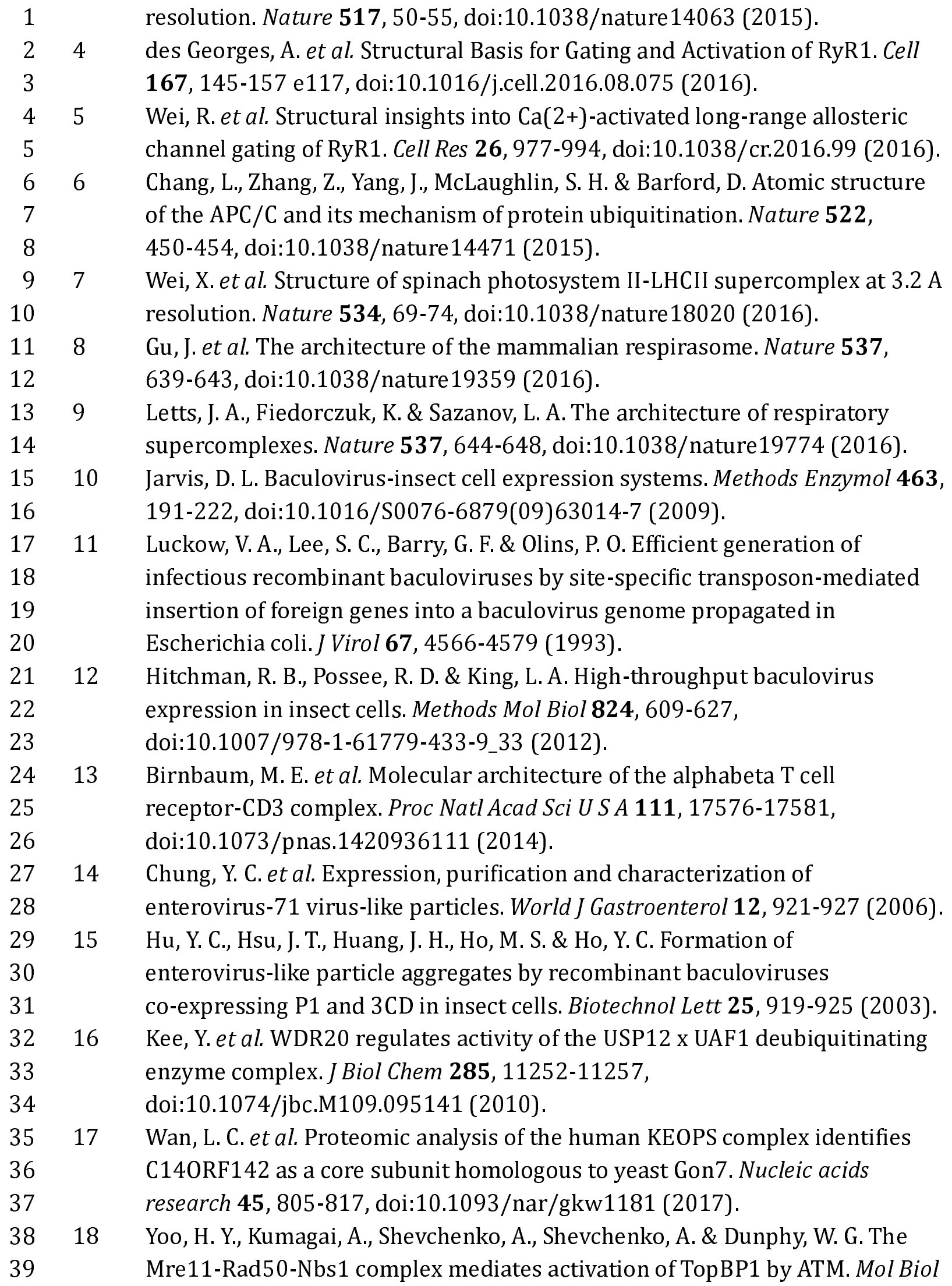


Cell 20, 2351-2360, doi:10.1091/mbc.E08-12-1190 (2009).

19 Belyaev, A. S. \& Roy, P. Development of baculovirus triple and quadruple expression vectors: co-expression of three or four bluetongue virus proteins and the synthesis of bluetongue virus-like particles in insect cells. Nucleic acids research 21, 1219-1223 (1993).

20 Berger, I., Fitzgerald, D. J. \& Richmond, T. J. Baculovirus expression system for heterologous multiprotein complexes. Nat Biotechnol 22, 1583-1587, doi:10.1038/nbt1036 (2004).

21 Bartlam, M., Yang, H. \& Rao, Z. Structural insights into SARS coronavirus proteins. Curr Opin Struct Biol 15, 664-672, doi:10.1016/j.sbi.2005.10.004 (2005).

22 Ziebuhr, J., Snijder, E. J. \& Gorbalenya, A. E. Virus-encoded proteinases and proteolytic processing in the Nidovirales. J Gen Virol 81, 853-879, doi:10.1099/0022-1317-81-4-853 (2000).

23 Sawicki, S. G., Sawicki, D. L. \& Siddell, S. G. A contemporary view of coronavirus transcription. J Virol 81, 20-29, doi:10.1128/JVI.01358-06 (2007).

24 Vijayachandran, L. S. et al. Robots, pipelines, polyproteins: enabling multiprotein expression in prokaryotic and eukaryotic cells. J Struct Biol 175, 198-208, doi:10.1016/j.jsb.2011.03.007 (2011).

25 Nie, Y., Bellon-Echeverria, I., Trowitzsch, S., Bieniossek, C. \& Berger, I. Multiprotein complex production in insect cells by using polyproteins. Methods Mol Biol 1091, 131-141, doi:10.1007/978-1-62703-691-7_8 (2014).

26 Liu, Q., Li, M. Z., Leibham, D., Cortez, D. \& Elledge, S. J. The univector plasmid-fusion system, a method for rapid construction of recombinant DNA without restriction enzymes. Curr Biol 8, 1300-1309 (1998).

27 Metcalf, W. W., Jiang, W. \& Wanner, B. L. Use of the rep technique for allele replacement to construct new Escherichia coli hosts for maintenance of R6K gamma origin plasmids at different copy numbers. Gene 138, 1-7 (1994).

28 Filutowicz, M. et al. Role of the pi initiation protein and direct nucleotide sequence repeats in the regulation of plasmid R6K replication. Basic Life Sci 30, 125-140 (1985).

29 Hill-Perkins, M. S. \& Possee, R. D. A baculovirus expression vector derived from the basic protein promoter of Autographa californica nuclear polyhedrosis virus. J Gen Virol 71 ( Pt 4), 971-976, doi:10.1099/0022-1317-71-4-971 (1990).

30 van Oers, M. M. Opportunities and challenges for the baculovirus expression system. J Invertebr Pathol 107 Suppl, S3-15, doi:10.1016/j.jip.2011.05.001 (2011). 
131 Ishiyama, S. \& Ikeda, M. High-level expression and improved folding of

proteins by using the vp39 late promoter enhanced with homologous DNA regions. Biotechnol Lett 32, 1637-1647, doi:10.1007/s10529-010-0340-7 (2010).

32 Li, S. F., Wang, H. L., Hu, Z. H. \& Deng, F. Genetic modification of baculovirus expression vectors. Virol Sin 27, 71-82, doi:10.1007/s12250-012-3236-y (2012).

33 Gibson, D. G. et al. Enzymatic assembly of DNA molecules up to several hundred kilobases. Nat Methods 6, 343-345, doi:10.1038/nmeth.1318 (2009).

34 Pijlman, G. P., van Schijndel, J. E. \& Vlak, J. M. Spontaneous excision of BAC vector sequences from bacmid-derived baculovirus expression vectors upon passage in insect cells. J Gen Virol 84, 2669-2678, doi:10.1099/vir.0.19438-0 (2003).

35 Wu, B. \& Guo, W. The Exocyst at a Glance. J Cell Sci 128, 2957-2964, doi:10.1242/jcs.156398 (2015).

36 Heider, M. R. et al. Subunit connectivity, assembly determinants and architecture of the yeast exocyst complex. Nat Struct Mol Biol 23, 59-66, doi:10.1038/nsmb.3146 (2016).

37 Reck-Peterson, S. L. Dynactin revealed. Nat Struct Mol Biol 22, 359-360, doi:10.1038/nsmb.3022 (2015).

38 Urnavicius, L. et al. The structure of the dynactin complex and its interaction with dynein. Science (New York, N.Y 347, 1441-1446, doi:10.1126/science.aaa4080 (2015).

39 Wang, S. et al. Structural characterization of coatomer in its cytosolic state. Protein Cell 7, 586-600, doi:10.1007/s13238-016-0296-z (2016).

40 Zhang, K. et al. Cryo-EM Reveals How Human Cytoplasmic Dynein Is Auto-inhibited and Activated. Cell 169, 1303-1314 e1318, doi:10.1016/j.cell.2017.05.025 (2017).

41 Mosadeghi, R. et al. Structural and kinetic analysis of the COP9-Signalosome activation and the cullin-RING ubiquitin ligase deneddylation cycle. Elife $\mathbf{5}$, doi:10.7554/eLife.12102 (2016).

42 Zheng, N. et al. Structure of the Cul1-Rbx1-Skp1-F boxSkp2 SCF ubiquitin ligase complex. Nature 416, 703-709, doi:10.1038/416703a (2002).

43 Berger, I. et al. The multiBac protein complex production platform at the EMBL. J Vis Exp, e50159, doi:10.3791/50159 (2013).

44 Chen, X., Pham, E. \& Truong, K. TEV protease-facilitated stoichiometric delivery of multiple genes using a single expression vector. Protein Sci 19, 2379-2388, doi:10.1002/pro.518 (2010). 
145 Bieniossek, C., Imasaki, T., Takagi, Y. \& Berger, I. MultiBac: expanding the

2 research toolbox for multiprotein complexes. Trends Biochem Sci 37, 49-57,

3 doi:10.1016/j.tibs.2011.10.005 (2012).

4

5 
2 Figure 1. SmartBac vector maps. The SmartBac system includes four acceptor

3 plasmids (4V1G, 4V1R, 5V1TG and 5V1TR) and two donor plasmids (4V2G and

4 4V2R). Vector maps were produced by SnapGene Software

5 (http://www.snapgene.com/).

6 Figure 2. Schemes for the expression of large multiprotein complexes. (a)

7 The eight-subunit protein complex to be expressed. The eight genes are divided

8 into two groups according to their sizes. Two long polyproteins are designed with

9 TEV cleavage sites separating the adjacent genes. $\Sigma$ represents the TEV

10 cleavage site. (b) Schematic representation of Scheme 1 for the expression of

11 multiprotein complexes with a molecular weight less than $600 \mathrm{kDa}$. Here the

12 acceptor vector $4 \mathrm{~V} 1 \mathrm{R}$ is used, but $5 \mathrm{~V} 1 \mathrm{TR}$ can also be used. (c) Schematic

13 representation of Scheme 2 for the expression of multiprotein complexes with a

14 molecular weight greater than $600 \mathrm{kDa}$. The fluorescent protein in the $4 \mathrm{~V} 2 \mathrm{G} / 4 \mathrm{~V} 2 \mathrm{R}$

15 donor vector is not expressed because a stop codon has been inserted at the end

16 of the fusion gene, which is located at the upstream of the coding sequence of the

17 fluorescent protein. The coding sequences of EGFP and tagRFP can also be

18 removed by restriction enzyme digestion.

19 Figure 3. Screening for the best affinity-tagged subunit through co-infection

20 of insect cells (Scheme 3). (a) Diagrams of the ten types of transfer plasmids.

21 The final transfer plasmids, ABCD (RFP) and EFGH (GFP), are generated using

22 Scheme 2, and each will express four protein subunits without affinity labels. Each

23 of the other eight transfer plasmids will express one subunit with an $\mathrm{N}$-terminal

24 Twin-Strep (TS) tag. Either the $4 \mathrm{~V} 1$ or $5 \mathrm{~V} 1$ vector can be used here. (b)

25 Production of ten types of recombinant baculoviruses (RBVs). Transformation of 
1 the ten types of plasmids into DH10Bac competent cells generates 10 types of

2 RBVs. (c) Screening baculovirus combinations to find the subunit that results in

3 the best purification. The ten types of RBVs are divided into eight groups, and

4 each group contains BV-ABCD (RFP), BV-EFGH (GFP) and one BV-TSn (where

$5 \mathrm{n}$ corresponds to the subunit, $\mathrm{A}$ to $\mathrm{H}$ ). Insect cells are co-infected with eight

6 groups of RBVs and strep-affinity resin is used to pull down proteins bound to the

7 TS-tagged subunit. The tagged subunit that allows the best purification of the

8 whole complex is selected. In this example, subunit $\mathrm{H}$ is the best. (d) Production

9 of the multiprotein complex. Based on the screening result in (c), a new final

10 transfer plasmid EFG-TSH (GFP) is constructed, which expresses an N-terminal

11 TS-tagged subunit $H$. The whole protein complex will be purified from insect cells

12 co-infected with BV-ABCD (RFP) and BV-EFG-TSH (GFP).

13 Figure 4 Examples of multiprotein complexes expressed using the

14 SmartBac system. (a) Fluorescence signals for tagRFP (top) and EGFP (bottom)

15 detected from Sf9 cells transfected with BVE1S547 and BV2863 (see Table 1). (b)

16 Coomassie-stained SDS-PAGE gel of human exocyst complex purified using

17 eight different Twin-Strep tagged subunits (BV-SE1 to BV-SE8, see Table 1). (c)

18 Coomassie-stained SDS-PAGE gel of human exocyst complex purified from

19 insect cells co-infected with BV-2863 and BV-E1S547 (see Table 1). The exocyst

20 complex was purified using Twin-Strep-tagged subunit EXOC5. (d) Electron

21 micrograph of negative-stained recombinant human exocyst complex. The bar

22 represents $100 \mathrm{~nm}$. (e) Representative classes from 2D classification of

23 recombinant human exocyst complex particles. (f) 3D reconstruction of

24 recombinant human exocyst complex based nsEM data. (g) Coomassie-stained

25 SDS-PAGE gel of the human dynactin complex purified by one-step strep-affinity

26 purification. (h) Coomassie-stained 3-8\% Native-PAGE gel of purified human 
1 dynactin complex after glycerol density gradient centrifugation purification. (i)

2 Single-particle nsEM analysis of recombinant human dynactin complex with the

3 representative raw micrograph (top) and 2D class averages (bottom). Scale bar,

$450 \mathrm{~nm}$. (j) Coomassie-stained SDS-PAGE gel of purified recombinant human

5 COPI complex, human dynein complex, human CSN complex and human SCF

6 complex.

7 
1 Table 1. Recombination of human Exocyst complex using SmartBac system

2

\begin{tabular}{|c|c|c|c|}
\hline Subunit & $\begin{array}{c}\text { Intermediate } \\
\text { plasmid }\end{array}$ & $\begin{array}{c}\text { Final } \\
\text { transfer } \\
\text { plasmid }\end{array}$ & $\begin{array}{c}\text { Recombinant } \\
\text { baculovirus }\end{array}$ \\
\hline $\begin{array}{c}\text { TS-tagged } \\
\text { EXOCn }\end{array}$ & 5V1TG-SEn & BV-SEn \\
\hline $\begin{array}{c}\text { EXOC2, } \\
\text { EXOC8 }\end{array}$ & $4 \mathrm{~V} 2-\mathrm{E} 28$ & E2863 & BV-E2863 \\
\hline $\begin{array}{c}\text { EXOC6, } \\
\text { EXOC3 }\end{array}$ & $5 \mathrm{~V} 1 \mathrm{TG}-\mathrm{E} 63$ & & \\
\hline $\begin{array}{c}\text { EXOC1, } \\
\text { EXOC5 }\end{array}$ & $4 \mathrm{~V} 2-\mathrm{E} 15$ & E1547 & BV-E1547 \\
\hline $\begin{array}{c}\text { EXOC4, } \\
\text { EXOC7 }\end{array}$ & 5V1TR-E47 & & \\
\hline $\begin{array}{c}\text { EXOC1, } \\
\text { TS-tagged } \\
\text { EXOC5 }\end{array}$ & 4V2-E1SE5 & E1S547 & BV-E1S547 \\
\hline
\end{tabular}

3

4

5 
1 Table 2. Recombination of human Dynactin complex using SmartBac

2 system

3

\begin{tabular}{|c|c|c|c|}
\hline Subunit & $\begin{array}{l}\text { Intermediat } \\
\text { e plasmid }\end{array}$ & $\begin{array}{l}\text { Final } \\
\text { transfer } \\
\text { plasmid }\end{array}$ & $\begin{array}{l}\text { Recombina } \\
\text { nt baculovirus }\end{array}$ \\
\hline $\begin{array}{l}\text { TS-tagg } \\
\text { ed p135 }\end{array}$ & \multirow{3}{*}{ 5V1TG-M5 } & \multirow{3}{*}{ 5V1TG-M5 } & \multirow{3}{*}{ BV-M5 } \\
\hline p50 & & & \\
\hline p24 & & & \\
\hline Arp1 & \multirow[b]{2}{*}{$4 \mathrm{~V} 2-\mathrm{A}$} & \multirow{8}{*}{$A B$} & \multirow{8}{*}{$B V-A B$} \\
\hline $\begin{array}{c}\text { Beta-acti } \\
\mathrm{n}\end{array}$ & & & \\
\hline $\begin{array}{l}\text { CapZ } \\
\text { alpha }\end{array}$ & \multirow{6}{*}{ 5V1TR-B } & & \\
\hline $\begin{array}{l}\text { CapZ } \\
\text { beta }\end{array}$ & & & \\
\hline $\begin{array}{l}\text { P25(DC } \\
\text { TN5) }\end{array}$ & & & \\
\hline $\begin{array}{l}\text { P27(DC } \\
\text { TN6) }\end{array}$ & & & \\
\hline Arp11 & & & \\
\hline $\begin{array}{l}\text { P62(DC } \\
\text { TN4) }\end{array}$ & & & \\
\hline
\end{tabular}




\section{Figure 1}
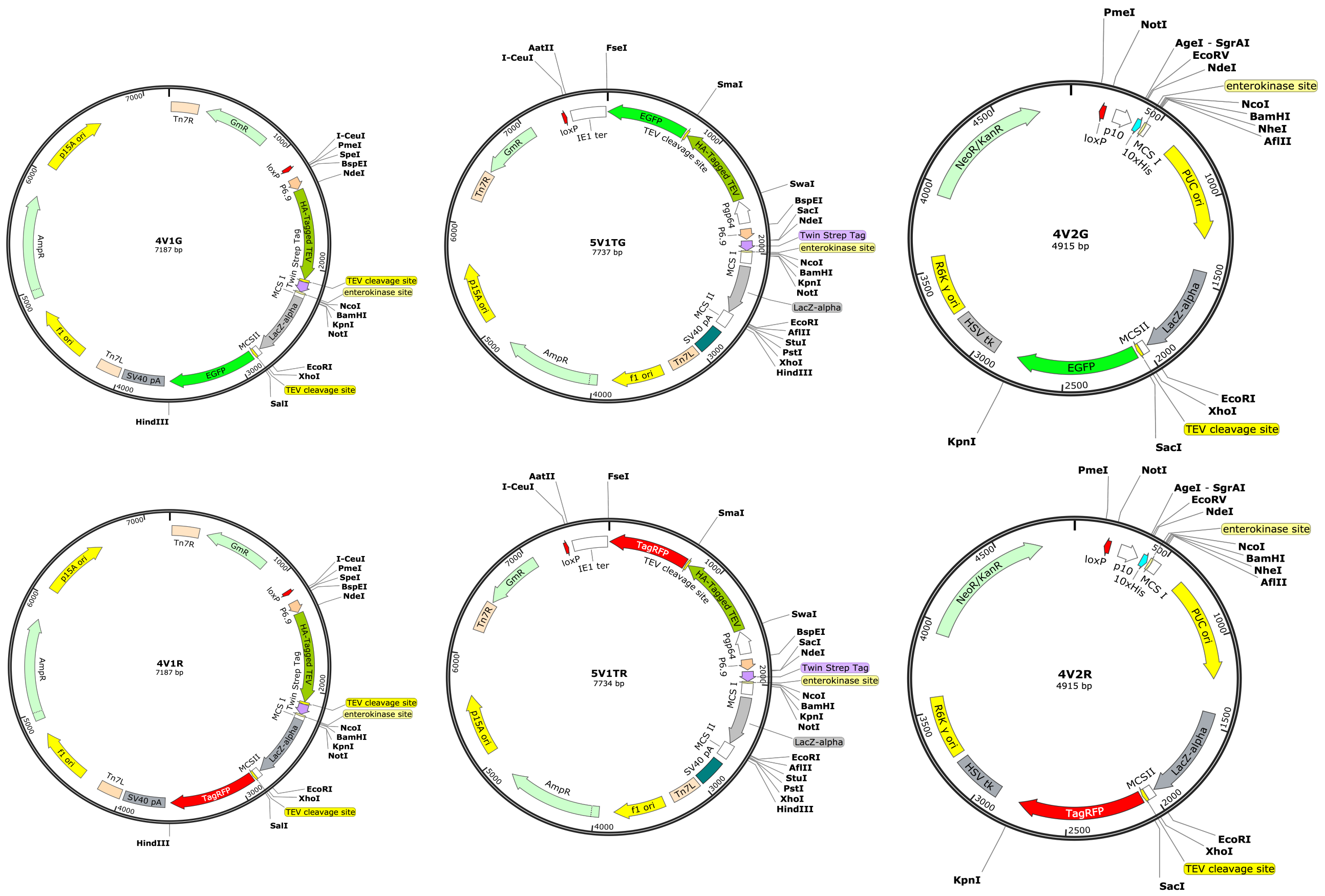


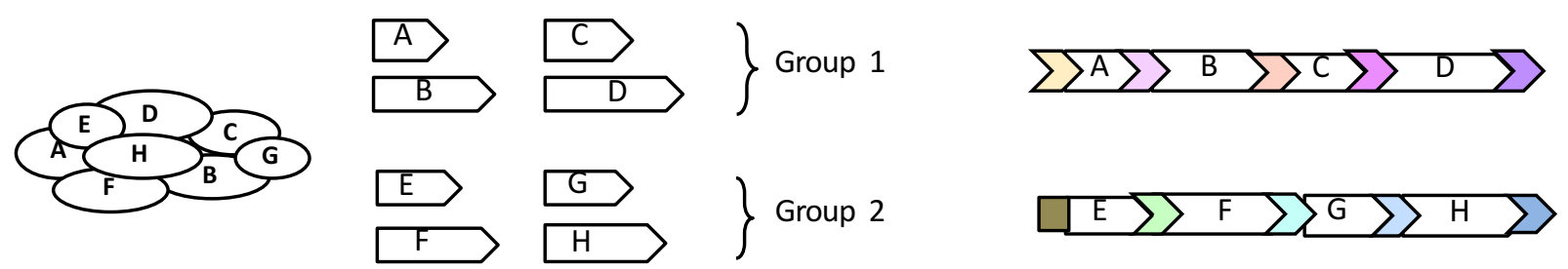

b
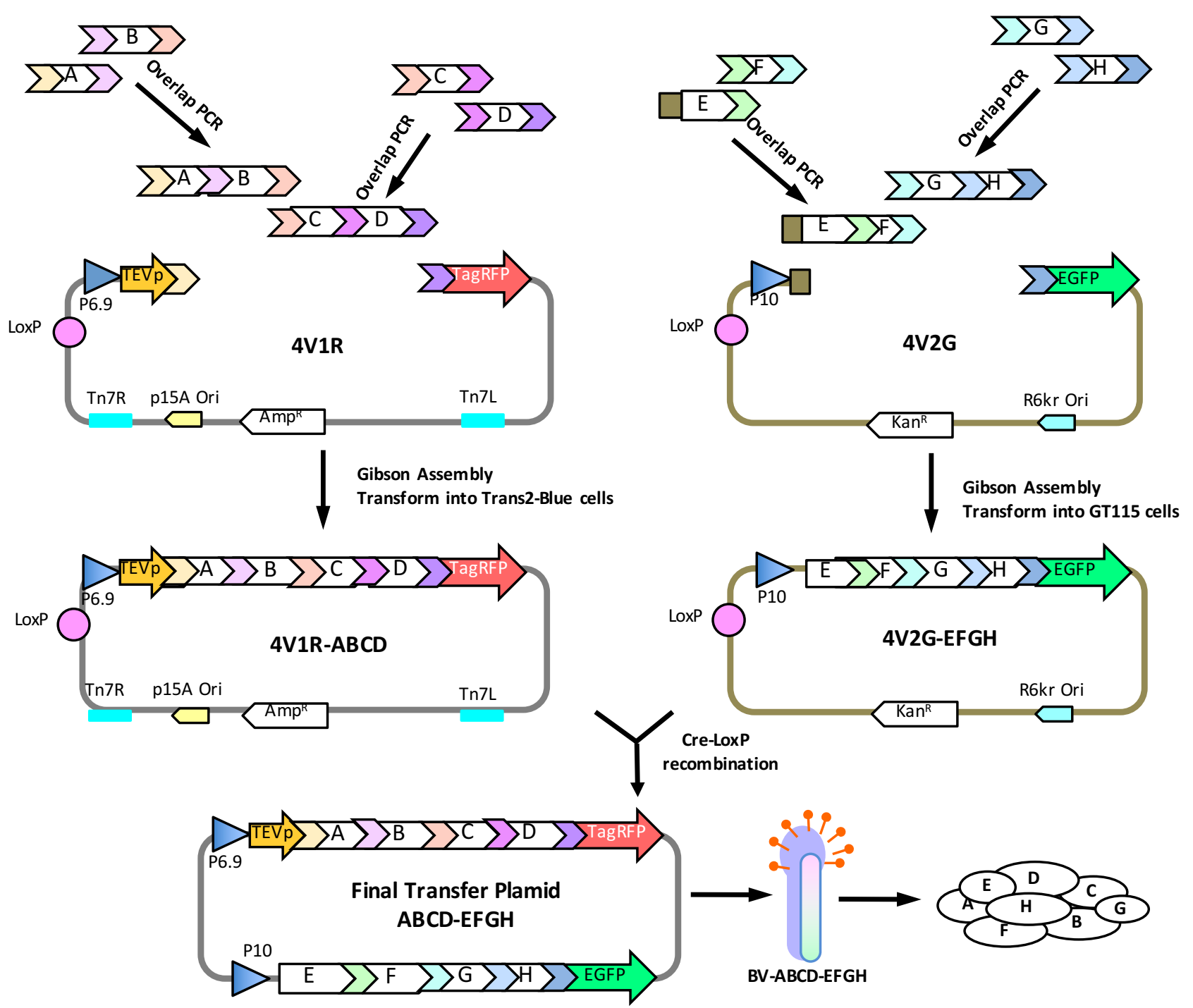


\section{Figure 2}

c

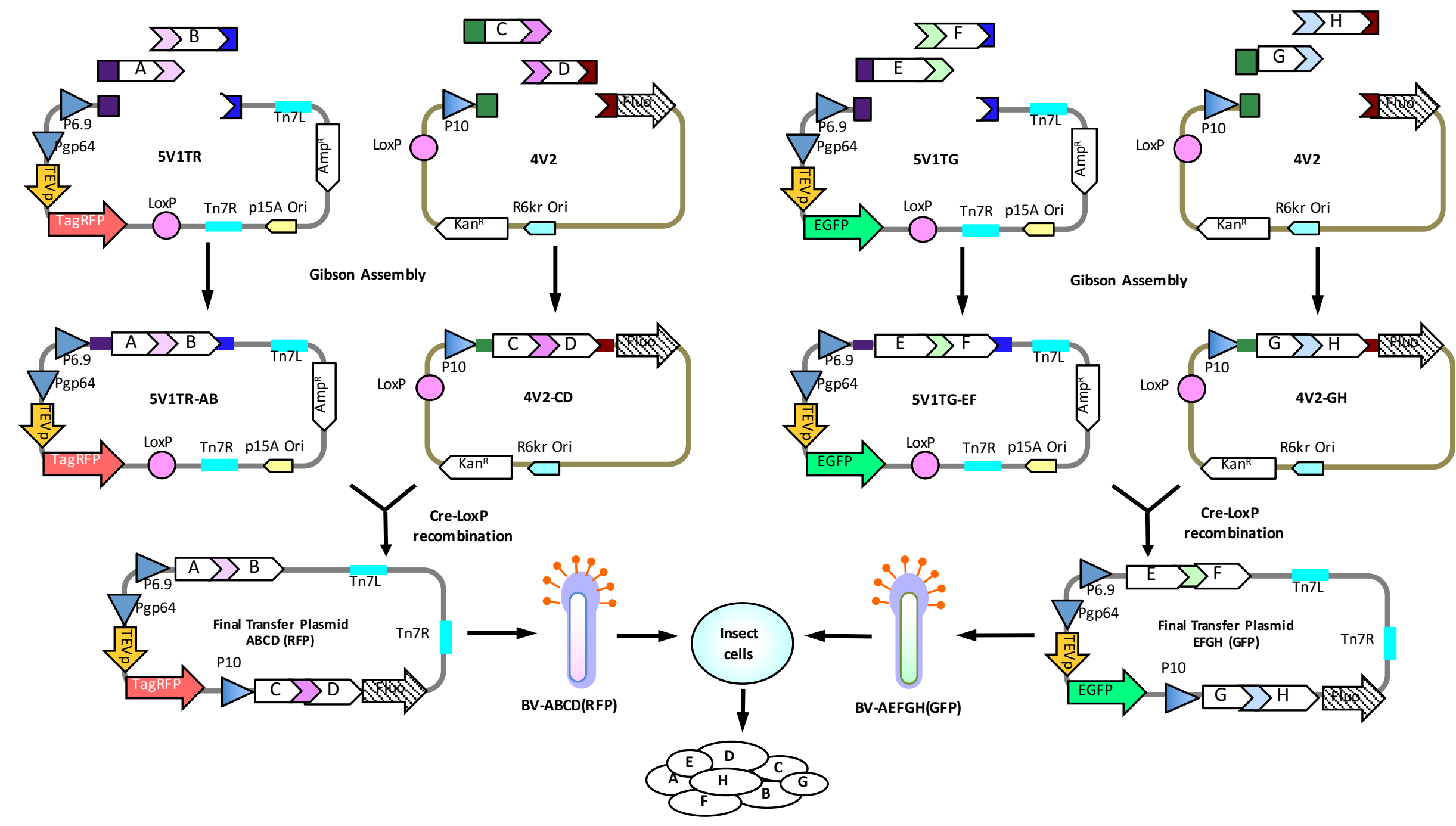




\section{Figure 3}

a
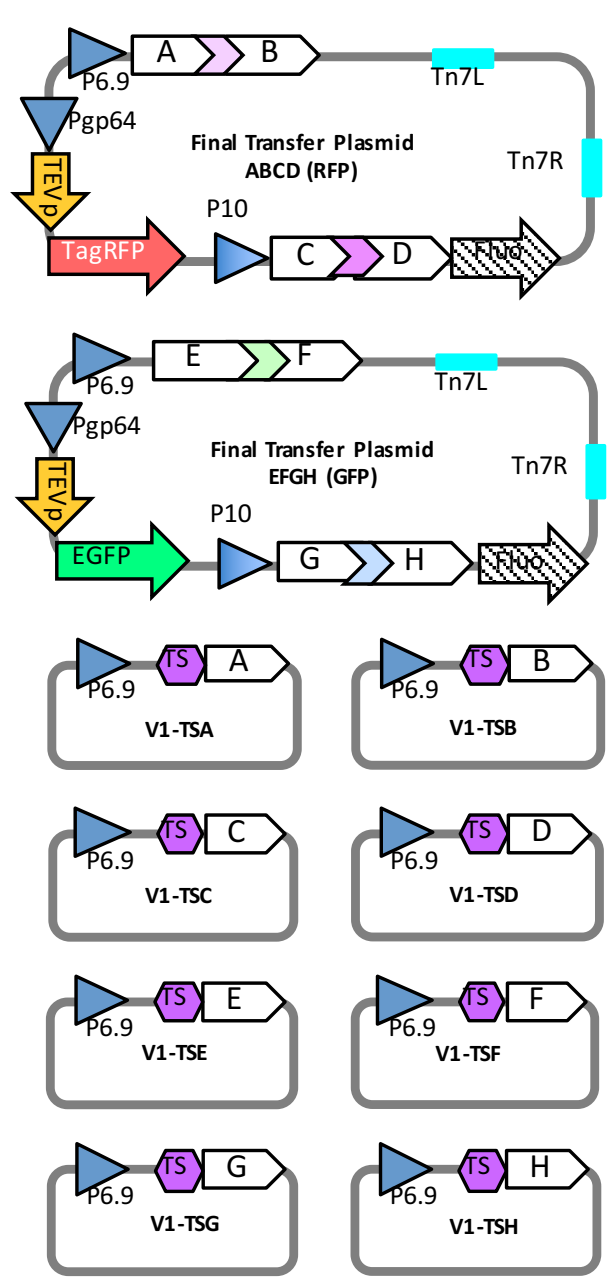

b
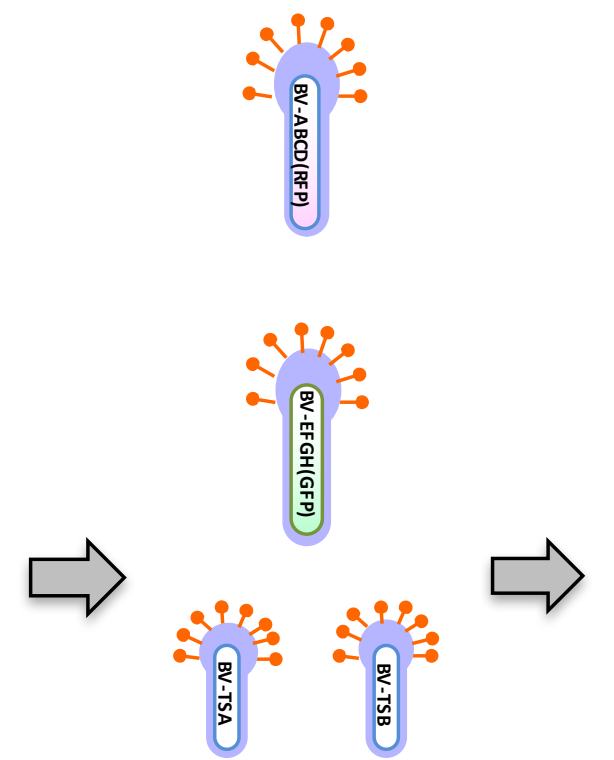

d

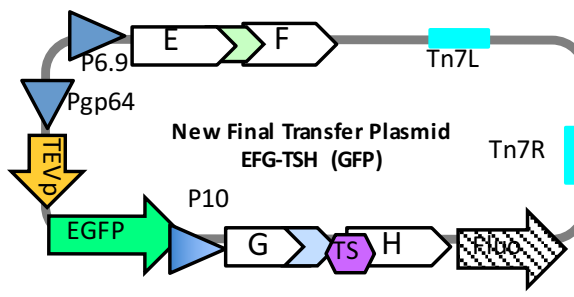

C

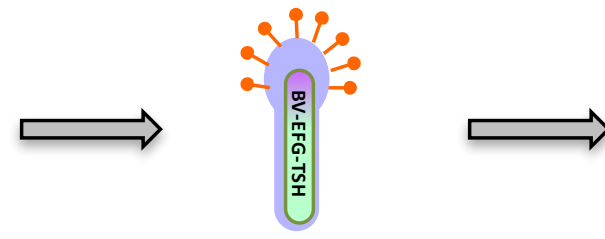

\begin{tabular}{|l|l|l|l|l|l|l|l|l|}
\hline & $\mathbf{1}$ & $\mathbf{2}$ & $\mathbf{3}$ & $\mathbf{4}$ & $\mathbf{5}$ & $\mathbf{6}$ & $\mathbf{7}$ & $\mathbf{8}$ \\
\hline Baculovirus 1 & TSA & TSB & TSC & TSD & TSE & TSF & TSG & TSH \\
\hline Baculovirus 2 & ABCD(RFP) \\
\hline Baculovirus 3 & EFGH(GFP) \\
\hline
\end{tabular}
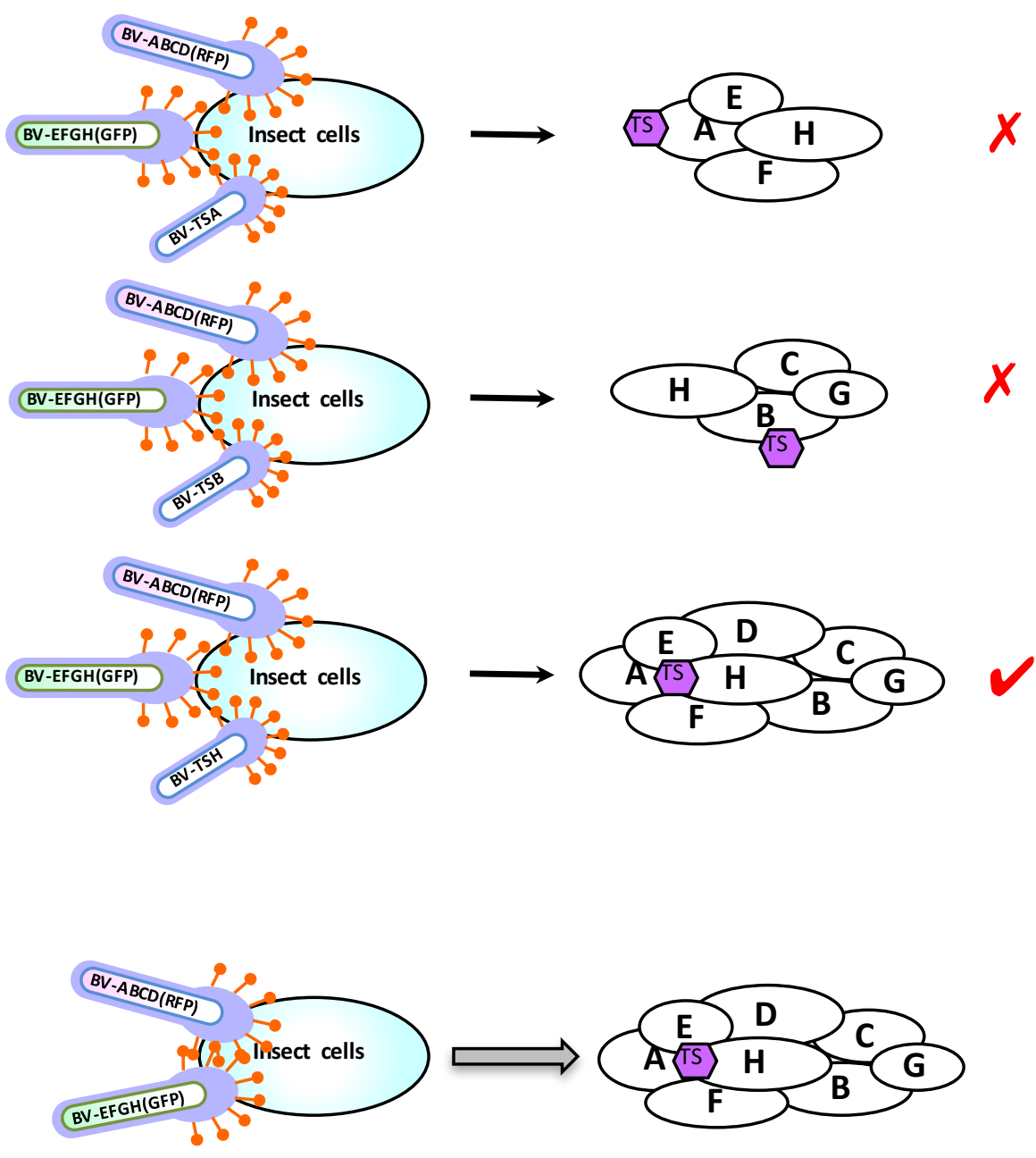
a
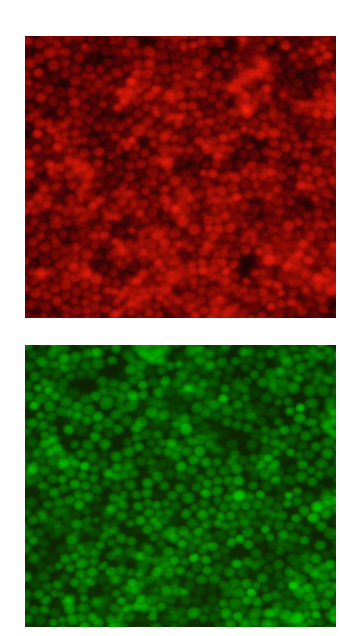

d

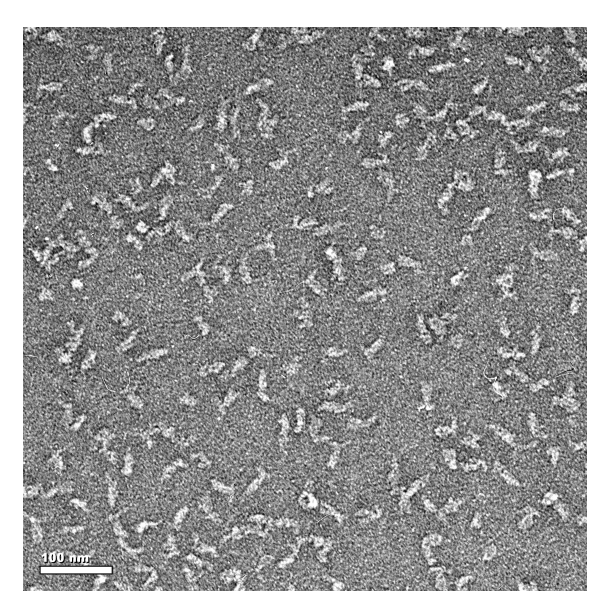

b

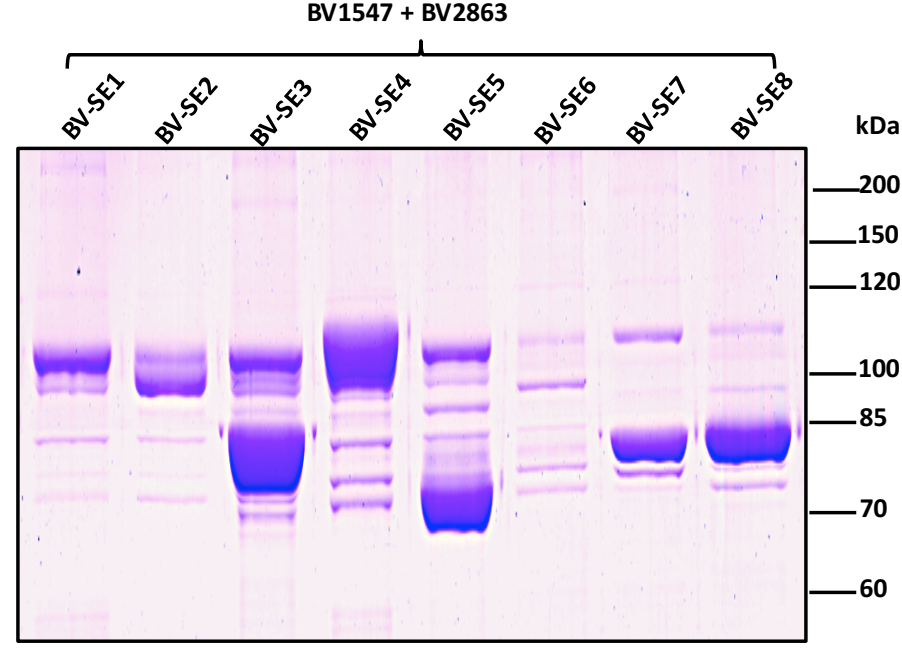

e

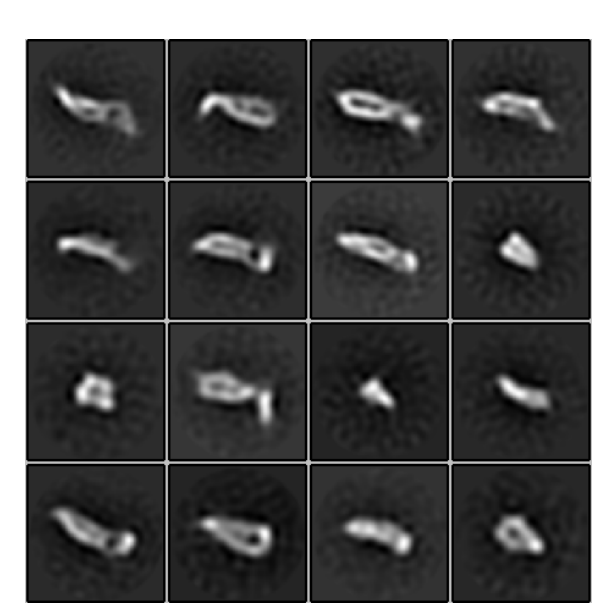

C Exocyst Complex

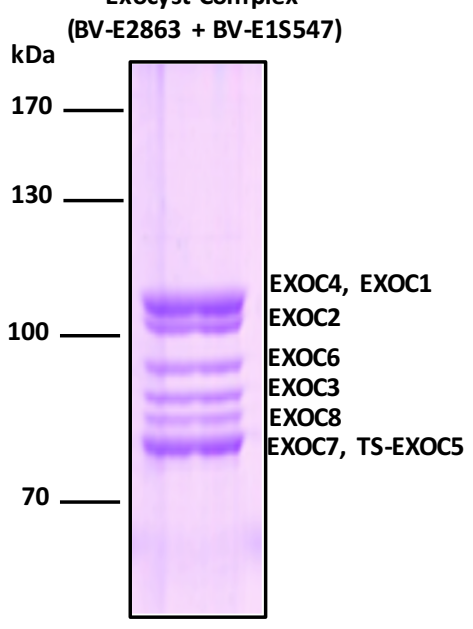

f

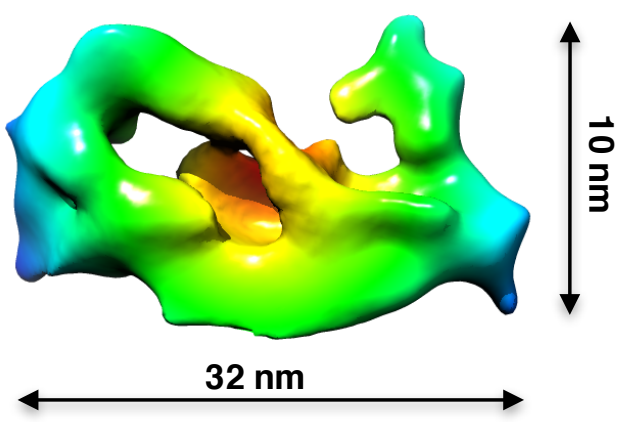




\section{Figure 4}

g

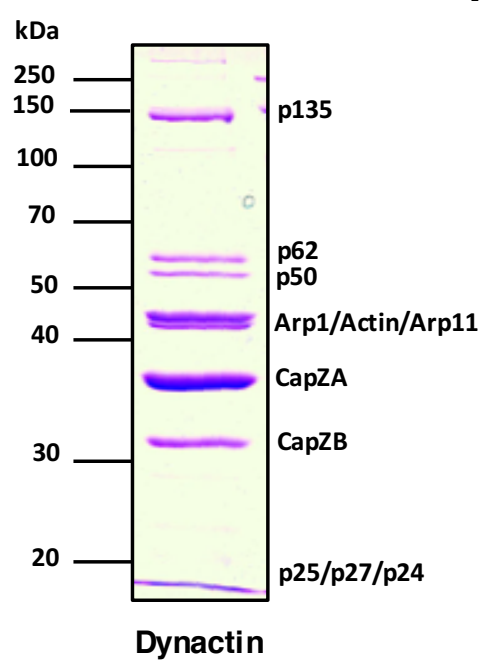

j

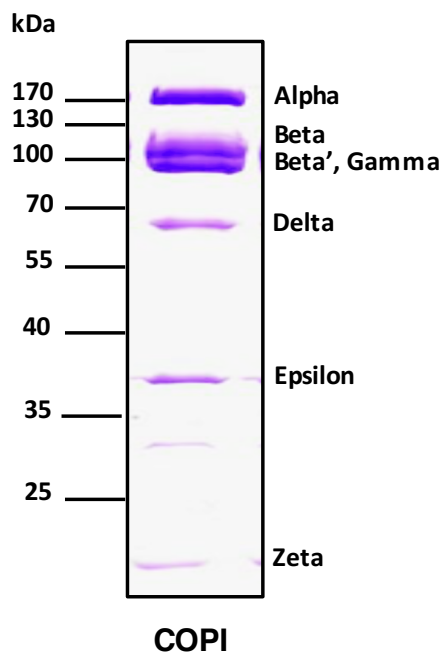

h

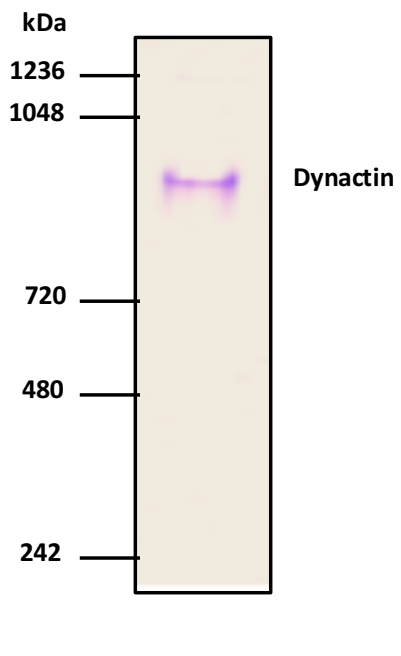

i

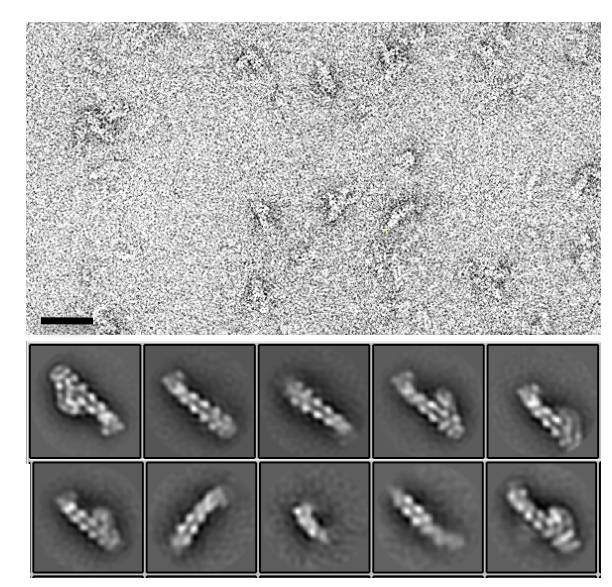

kDa

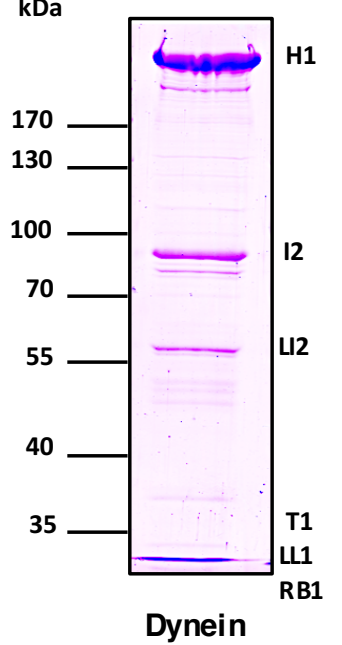

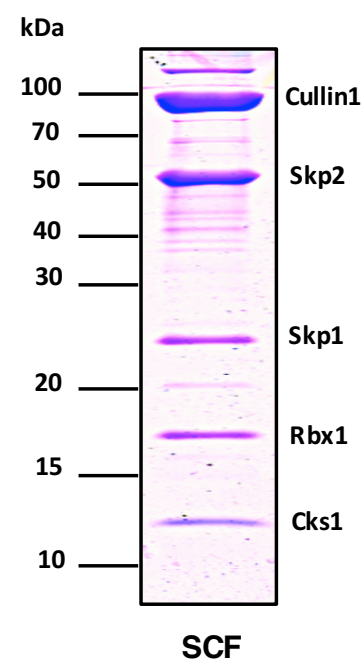

